\title{
Skills-Training in Gruppen ist gut wirksam
}

Fragestellung: Welche Komponenten der dialektisch-behavioralen Therapie (DBT) sind für deren Wirksamkeit bei der Borderline-Persönlichkeitsstörung (BPS) verantwortlich?

Hintergrund: Die DBT nach Marsha Linehan ist eine wirksame Psychotherapie zur Behandlung suizidaler Patienten mit BPS [1]. Hauptkomponenten der Therapie sind Einzeltherapie, Gruppen-Skills-Training, Telefon-Coaching sowie Supervision. Bisher ist unklar, welche der Therapieelemente zu einem positiven Therapieergebnis führen. Hier wurde untersucht, welche Rolle dabei das Gruppen-Skills-Training spielt.

Patienten und Methodik: Die Autoren führten zwischen 2004 und 2010 eine randomisierte kontrollierte Therapiestudie durch, in die 99 Frauen mit BPS (mittleres Alter 30,3 Jahre) eingeschlossen wurden, die mindestens zwei Suizidversuche und/ oder nicht suizidale Selbstverletzungen in den letzten fünf Jahren zeigten, einen Suizidversuch im letzten Jahr und mindestens einen Suizidversuch oder eine Selbstverletzung in den acht Wochen vor Screening hatten. Die Patientinnen wur-

Linehan MM, Korslund KE, Harned MS et al. Dialectical behavior therapy for high suicide risk in individuals with borderline personality disorder: a randomized clinical trial and component analysis. JAMA Psychiatry 2015; 72: $475-82$ den randomisiert auf eine der drei Behandlungsgruppen zugewiesen: Skills-Training plus „case management“ (DBT-S), individuelle DBT plus Aktivitätengruppe (DBT-I) und Standard-DBT, also individuelle DBT plus
Skills-Training (DBT). Die Behandlungen erfolgten über ein Jahr mit einjähriger Katamnese. Die Therapiedosis war in allen drei Armen gleich hoch und alle Gruppen verwendeten das DBT-Behandlungs-Manual zur Einschätzung und zum Management von Suizidalität. Die Therapieeffekte wurden dreimonatlich gemessen, wobei das primäre Wirkkriterium die Häufigkeit und Schwere von Suizidversuchen beziehungsweise Selbstverletzungen war. Die Haupthypothese war, dass Standard-DBT den beiden anderen Verfahren überlegen ist.

Ergebnisse: Alle drei Gruppen führten zu ähnlichen Verbesserungen in den Hauptzielparametern. Im Vergleich zur DBT-I führten während der einjährigen Behandlungsphase die beiden Gruppen, die Skills-Training beinhalteten, zu größeren Verbesserungen bei Selbstverletzungen (F1,85 $=59,1[\mathrm{p}<0,001]$ für DBT und F1,85 = 56,3 [p < 0,001] für DBT-S) und depressiven Symptomen $(\mathrm{t} 399=1,8[\mathrm{p}=0,03]$ für DBT und t399 = 2,9 [p = 0,004] für DBT-S). Zudem verbesserten sich Angstsymptome nur in diesen beiden Therapiearmen, aber nicht in der DBT-I-Gruppe. Standard-DBT führte gegenüber der DBT-I-Behandlung zu niedrigeren Dropout-Raten ( 8 vs. 16 Patienten) und zu weniger Kriseninterventionen während des Katamnesezeitraums.

Schlussfolgerungen: Verschiedene DBT-Interventionen, die das DBT-Therapiemanual verwenden, sind in der Reduktion von Suizidversuchen und Selbstverletzungen wirksam. Interventionen, die Skills-Training beinhalten, scheinen effektiver zu sein. Unterschiede in der Wirksamkeit zwischen Standard-DBT und DBT-S zeigen sich nicht.

\section{Wichtig für die flächendeckende Implementierung der DBT}

Die Studie erfüllt hohe methodische Qualitätskriterien und beantwortet eine sehr zentrale Frage in der Behandlung der BPS, nämlich ob ein Skills-Training mit begleitendem "clinical management " einer Standard-DBT in zwei Patientenrelevanten Outcomes, nämlich der Reduktion von Suizidversuchen und Selbstverletzungen überlegen ist. Diese Überlegenheit konnte nicht bestätigt werden. Was bedeutet das nun? Das Ergebnis ist zunächst einmal ein erster Hinweis, dass Skills-Training eine entscheidende Wirkkomponente der DBT ist. Unterstützt wird dieser Befund durch eine ältere Studie, die eine gute Kurzzeitwirksamkeit von Skills-Training auf verschiedene Symptome wie Ärger, affektive Instabilität, Impulsivität, Depressivität und Angst zeigen konnte [2]. Zweitens ist der Befund relevant, weil er mit einem Dogma der DBT aufräumt, dass nämlich nur die Kombination aus Einzel- und Gruppen-DBT wirksam ist. Hier gilt jedoch einschränkend, dass bisher nur eine Wirkkomponentenstudie vorliegt. Das Ergebnis muss also noch in weiteren Studien bestätigt werden. Drittens ist der Befund für die flächendeckende Versorgung mit DBT sehr wichtig. Bisher kann Standard-DBT mangels qualifizierter Therapeuten und dem hohen Aufwand in vielen Regionen in Deutschland nicht angeboten werden. Da aber bis zu acht Patienten in DBT-SkillsGruppen behandelt werden können, kann durch den Ausbau von ambulanten Skills-Gruppen die Versorgungssituation von BPS-Patienten nun entscheidend verbessert werden. Und dies realistischerweise auch dadurch, dass das „clinical management " im DBT-S-Arm darin bestand, dass die Patientinnen minimal einmal pro Monat einen persönlichen oder Telefonkontakt mit dem Therapeuten und maximal einmal pro Woche einen persönlichen Kontakt hatten und in diesen Kontakten psychoedukativ gearbeitet, ressourcenorientiert Probleme gelöst und suizidale Krisen gemanagt wurden. Dies lässt sich durchaus in der aktuellen Versorgungssituation in Deutschland realisieren.

\footnotetext{
Referenzen:

1. Stoffers JM et al. Cochrane Database Syst Rev 2012; 8: CD005652

2. Soler J et al. Behav Res Ther 2009; 47: $353-8$
} 